

\title{
La crise des relations professionnelles en Allemagne de l'Est et l'évolution de la négociation paritaire allemande François Bafoil
}

\section{To cite this version:}

François Bafoil. La crise des relations professionnelles en Allemagne de l'Est et l'évolution de la négociation paritaire allemande. Politix, 1996, 9 (33), pp.87-110. 10.3406/polix.1996.1939 . hal01010105

\section{HAL Id: hal-01010105 \\ https://hal-sciencespo.archives-ouvertes.fr/hal-01010105}

Submitted on 19 Jun 2014

HAL is a multi-disciplinary open access archive for the deposit and dissemination of scientific research documents, whether they are published or not. The documents may come from teaching and research institutions in France or abroad, or from public or private research centers.
L'archive ouverte pluridisciplinaire HAL, est destinée au dépôt et à la diffusion de documents scientifiques de niveau recherche, publiés ou non, émanant des établissements d'enseignement et de recherche français ou étrangers, des laboratoires publics ou privés. 


\section{La crise des relations professionnelles en Allemagne de l'Est et l'évolution de la négociation paritaire allemande}

In: Politix. Vol. 9, N³3. Premier trimestre 1996. pp. 87-110.

\section{Résumé}

La crise des relations professionnelles en Allemagne de l'Est et l'évolution de la négociation paritaire allemande.

François Bafoil [87-110].

Le transfert centralisé des institutions ouest-allemandes en Allemagne de l'Est a provoqué l'émergence de nombreux obstacles. Dans le cas des relations professionnelles, les règles de la négociation collective ont subi de profondes modifications, infléchissant ainsi la nature néo-corporatiste du jeu institutionnel allemand. Cela a tenu autant à la nature des partenaires sociaux, qu'à la situation des entreprises, ou encore au contenu des négociations. En dépit des difficultés, les syndicats sont apparus comme un acteur social innovant de premier plan, et les dérégulations qui ont vu le jour localement en Allemagne de l'Est ont préfiguré celles prônées à l'Ouest pour l'Allemagne entière. Le débat initié dans les années quatre-vingt concernant le niveau pertinent de la négociation collective s'est trouvé ainsi relancé, mais selon des modalités particulières où la branche semble pouvoir de moins en moins jouer un rôle homogénéisateur.

\section{Abstract}

The Industrial Relations Crisis in East Germany and the Evolution of German Joint Negotiation.

François Bafoil [87-110].

The centralized transfer of West German institutions to East Germany provoked the emergence of numerous obstacles. In the case of industrial relations, the rules of collective negotiation have been profoundly modified, lowering the neo-corporist nature of the German institutional game. This is due to the nature of the industrial actors, to the situations of the companies, and the content of the negotiations. Despite the obstacles, the unions have been a major innovating social actor, and the deregulations that have taken place locally in East Germany have prefigured those commended in the West part of Germany as a whole. The debate initiated in the 80 's about the right level of collective negotiation has therefore been revived, but under new modalities.

Citer ce document / Cite this document :

Bafoil François. La crise des relations professionnelles en Allemagne de l'Est et l'évolution de la négociation paritaire allemande. In: Politix. Vol. 9, N³3. Premier trimestre 1996. pp. 87-110.

doi : 10.3406/polix.1996.1939

http://www.persee.fr/web/revues/home/prescript/article/polix_0295-2319_1996_num_9_33_1939 


\title{
La crise des relations professionnelles en Allemagne de l'Est et l'évolution de la négociation paritaire allemande
}

\author{
François Bafoil \\ Centre de recherche sur le politique, \\ l'administration et le territoire \\ Institut d'études politiques de Grenoble
}

$\mathbf{P}$

OUR l'analyse des changements en Allemagne de l'Est, la théorie du transfert institutionnel ${ }^{1}$ présente un triple intérêt. Le premier est d'insister sur le caractère centralisé des procédures de transfert des règles ouest-allemandes, dont l'un des effets est de conduire à l'émergence de blocages en Allemagne de l'Est. Leur résolution traduit les capacités d'apprentissage des acteurs de l'Ouest, voire de l'Est. On a pu le vérifier avec le cas de la réforme administrative ou celle de l'entreprise industrielle, l'extension des normes techniques ou encore des relations contractuelles ayant entraîné la production de spirales de blocages ${ }^{2}$. Le second intérêt tient à la mise en valeur du poids des modèles de références, mentales et pratiques, pour des acteurs ouest-allemands insérés dans un tissu de relations institutionnelles fiable mais peu innovant en raison de sa densité $^{3}$. Le dernier trait renvoie à la possibilité de résoudre le paradoxe de la rupture d'un côté, initiée par le bouleversement de 1989-1990, et de la continuité, de l'autre, sensible au niveau de la reproduction des équilibres allemands dans la partie Est de l'Allemagne. C'est ainsi que certains changements ont pu être repérés localement comme autant d'*anomalies", dans le secteur agricole par exemple ${ }^{4}$, mais sans que le système général des règles en ait été affecté. Aux changements phénotypiques ont été opposées les permanences du génotype 5 .

L'examen du champ des relations professionnelles, durant la période de l'unification, apporte certaines inflexions à cette approche. En raison des

1. Lehmbruch (G.), -Die improvisierte Vereinigung : die dritte deutsche Republik•, Leviathan, 18, 1990 ; du même auteur .Institutionstransfer. Zur politischen Logik der Verwaltungsintegration in Deutschland, in Benz (A.), Seibel (W.), Mäding (H.), dir., Verwaltungsreform und verwaltungspolitik im Prozess der deutschen Vereinigung, Baden-Baden, Nomos Verlag, 1992, -Die deutsche Vereinigung, Strukturen der Politikentwicklung und strategische Anpassungsprozesse, in Kohler-Koch (B.), dir., Staat und Demokratie in Europa, Opladen, Westdeutscher Verlag, 1992 ; Seibel (W.), -Necessary Illusions. The Transformation of Governance Structures in the new Germany., La Reure Tocqueville, 13, 1992.

2. Voskamp (U.), Wittke (V.), -Aus Modernisierungsblokaden werden Abwärtsspiralen. Zur 2. Voskamp (U.), Wittke (V.), AUs Motrieben und Kombinaten der ehemaligen DDR., SOFI-Mitteilungen, 1991.

3. Czada (R.), Schleichweg in die "Dritte Republik". Politik der Vereinigung und politischer Wandel in Deutschland., Politische Vierteljabresscbrift, 35, 1994.

4. Lacquement $(G$.$) , :Les changements de modèle agricole dans les nouveaux Länder allemands.,$ La revrie d'études comparatives Est/Onest, 1993.

5. Robischon (T.), Stucke (A.), Wasem (J.), Wolf (H.G.), .Die politische Logik der deutschen Vereinigung und der Institutionstranfer : Eine Untersuchung am Beispiel von Gesundswesen, Forschungssystem und Telekommunikation,, Politisches Vierteljabresschrift, 36, 1995. 
dysfonctionnements qui ont vu le jour à la suite du transfert des règles, la nature néo-corporatiste du jeu institutionnel, mais également les fonctions assignées aux organisations tant syndicales que patronales dans le cours de l'unification, ont, en effet, été modifiées. Si l'on suit l'approche de Gerhard Lehmbruch, c'est l'intégration verticale et les procédures de concertation entre les organisations représentatives d'employeurs et d'employés qui soulignent le caractère corporatiste d'un État, autrement dit sa capacité d'inclusion et de mise en correspondance des objectifs sociétaux aux buts de l'organisation ${ }^{1}$. Ainsi, le degré de coalition des intérêts particuliers par des organisations représentatives peut fonder la croissance économique et garantir un bas niveau de conflits. Plus une organisation est centralisée, plus il lui appartient de contrôler les conséquences de son action et plus il lui est permis de peser sur les choix centraux, en faisant siens les objectifs généraux. Cependant les risques de efree rider sont plus élevés parmi ces grandes organisations que parmi les petites. Chez ces dernières en effet, la capacité de mobilisation des adhérents est plus forte et plus grand le contrôle interne sur leurs membres ${ }^{2}$. Par ailleurs, si les deux logiques de l'intégration, d'un côté, et de l'influence sur les choix publics, de l'autre, se combinent mieux dans les grandes organisations, il arrive qu'elles entrent en conflit. La logique de corps (Mitgliedschaftslogik) par laquelle l'organisation cherche à accroître ses potentialités grâce à la mobilisation de ses adhérents peut se révéler, dans certains cas, non complémentaire de la logique d'influence (Einflusslogik) par laquelle l'organisation cherche à conserver la marge qui est la sienne dans le jeu institutionnel en pesant au maximum sur les décisions finales. Or, c'est justement cette tension entre les deux logiques qui a puissamment pesé sur les acteurs des relations professionnelles allemandes, syndicats et patronat, durant l'unification, et conduit à l'élaboration de compromis.

Par ailleurs, les charges qui leur ont été assignées ont été affectées de profonds dysfonctionnements dès lors qu'elles ont été confrontées aux réalités issues de l'effondrement de la RDA. Ces agouvernements d'intérêts privés. ${ }^{3}$ que sont les organisations professionnelles ont eu, en effet, pour tâche d'assurer la reproduction des équilibres propres au jeu corporatiste ouest-allemand, d'intégrer les individus en leur sein et, enfin, d'assurer la production d'une identité collective par l'adhésion aux valeurs démocratiques propres à ces systèmes intermédiaires. La valeur de la citoyenneté a été placée au premier plan dans le Traité d'union allemande, entraînant avec elle la nécessité d'égaliser les conditions de vie et donc de remettre à niveau l'Allemagne de l'Est dans les plus brefs délais. La négociation collective sur les salaires devait être le moyen de cette égalisation et les partenaires sociaux, ses vecteurs. Or, de profonds déséquilibres ont contribué à modifier sensiblement le jeu institutionnel. Ils tiennent autant à l'absence prolongée du

1. Lehmbruch (G.), -Introduction : Néo-Corporatism in Comparative Perspective, in Lehmbruch (G.), Schmitter (P.C.), eds, Patterns of Corporatist Policy-Making, Londres, Sage, 1982. Contestant la validité du terme de corporatisme- et sa capacité a bien décrire la situation allemande, $K$. von Beyme préfère parler, quant à lui, de système de concertation entre les différentes organisations et associations, chargées de représenter la pluralité des intérêts sociaux professionnels ou politiques. Au delà de leurs différences d'approche théorique, les auteurs sont d'accord pour constater que les institutions organisées, tant politiques que sociales, constituent un des éléments de la permanence de l'organisation allemande, en dépit des différents régimes politiques depuis
un siècle.

2. Olson (M.), Logique de l'action collective, Paris, PUF, 1978.

3. Streeck (W.), Schmitter (P.), Communauty, Market, State, and Associations ? The Prospective Contribution of Interest Governance to Social Orders, in Streeck (W.), Schmitter (P.C.), eds Private Interest Govermment bejond Market and State, Londres, Sage, 1985. 
partenaire patronal, qu'aux attentes manifestées de part et d'autre de l'Allemagne, ou encore à la disjonction des éléments propres au compromis social. Ce dernier repose sur la production de standards sociaux élevés, en échange de taux de productivité économiques qui le sont également, mais qui dans la période de l'unification, n'ont pas vu le jour à l'Est. La crise estallemande a ainsi mis en lumière les limites du système de négociation au fondement de l'équilibre allemand. Plus encore, elle a aiguisé les tensions entre partenaires et réactivé les oppositions non résolues dans la décennie quatre-vingt, portant notamment sur le niveau pertinent de la négociation, qu'il s'agisse de l'entreprise, privilégiée par les représentants patronaux, ou bien de la branche, à laquelle tiennent fermement les acteurs syndicaux.

Ainsi, le terme de sstratégie du détour", qui a pu caractériser la politique de la Treubandanstalt ${ }^{1}$, peut également s'appliquer aux organisations syndicales, pour désigner les procédures mises en œuvre pour contourner et résoudre les obstacles nés de l'extension et de l'application unilatérale des règles de fonctionnement ouest-allemandes à la réalité est-allemande. Le résultat final loin de donner lieu à l'élargissement des capacités initiales, a plutôt débouché sur un approfondissement de la crise antérieure. En mettant au premier plan la question de la citoyenneté allemande pour des acteurs qui en ont été privés durant plus de cinquante ans, la crise est-allemande a masqué provisoirement les interrogations syndicales ouest-allemandes des années quatre-vingt, portant sur le niveau d'action ou sur les formes d'organisation pertinents. Loin de se trouver résolues par l'unification allemande, ces questions sont revenues avec un poids accru, dès lors que la récession s'est imposée en 1993 à l'ensemble de l'Allemagne, appelant la reformulation du compromis salarial et la redéfinition des contenus de la négociation.

L'objectif de cet article est d'analyser comment la dynamique du transfert institutionnel centralisé s'est trouvée entravée par les réalités est-allemandes, impliquant la disjonction des logiques d'action propres aux organisations partenaires du jeu, les conduisant à la reformulation de nombreux compromis nationaux et locaux, nécessaires à la poursuite du jeu. Dans cette dynamique d'où a fait défaut initialement le partenaire patronal, les syndicats se sont avérés une force innovatrice de premier plan, tant lors de la crise des accords salariaux qu'à l'occasion des politiques d'emploi et de développement économique. Certains éléments de flexibilité qui ont vu le jour en Allemagne de l'Est ont préfiguré ceux prônés à une plus grande échelle à l'Ouest, et valant pour l'Allemagne unie.

\section{La réunification de la représentation des intérêts}

À bien des égards, le transfert de l'action institutionnelle au niveau des relations professionnelles a entraîné l'apparition de nombreux blocages, soit que les réponses antérieures se soient révélées inadaptées au nouveau contexte, soit que les attentes des adhérents des différentes organisations aient été par trop divergentes, soit enfin que certains acteurs aient été tout simplement manquants. Dans ces conditions, le transfert institutionnel n'a pu

\footnotetext{
1. Bafoil (F.), .La Treuhandanstalt, un apprentissage organisationnel-, Politiques et Management Public, 1, 1996 ; Czada (R.), -Die Treuhandanstalt im Umfeld von Politik und Verbänder*, in Fischer (W.), Hax (H.), Schneider (H. K.), dir., Treubandanstalt : Das Unmögliche Wagen, Berlin, Akademie-Verlag, 1993.
} 
qu'être imparfait, et les solutions trouvées dans le cadre des négociations, insatisfaisantes.

\section{Les syndicats}

En dépit d'une adhésion rapide à compter du mois de février 1990 des membres et des fédérations de l'ancienne confédération syndicale estallemande (FDGB) aux syndicats appartenant à la confédération ouestallemande $(D G B)^{1}$, de nombreuses difficultés sont apparues, entravant l'adhésion des membres à la politique de l'organisation, et par conséquent la capacité d'action de cette dernière. La tension entre la capacité de mobilisation et la capacité de participation au jeu institutionnel central a entraîné de profonds déséquilibres. Tandis que les organisations syndicales pouvaient immédiatement imposer le contenu de la négociation paritaire aux représentants patronaux en raison, notamment, de leur légitimité numérique, mais également des attendus du Traité d'union en matière de citoyenneté, ils ne pouvaient pas, en revanche, prétendre le fonder sur la mobilisation des adhérents autour des enjeux de la négociation ouest-allemande. Ils le pouvaient d'autant moins qu'au processus centralisé de transfert des organisations et des règles du jeu institutionnel n'a pas correspondu une prise en charge centralisée des attentes exprimées par les acteurs est-allemands. Compte tenu des formes d'action et d'organisation prévalant à l'Ouest, il était attendu de la part des citoyens est-allemands, à la fois une acceptation passive des règles du jeu institutionnel, ce qui s'est globalement opéré, et une participation active aux actions décentralisées, ce qui a entraîné nombre de malentendus. D'emblée, les attentes et les représentations ont reflété d'importantes divergences de part et d'autre de l'Allemagne.

La première de ces difficultés est apparue alors même que l'édifice syndical du DGB ne s'était pas complètement reproduit dans la partie est de la nouvelle Allemagne ${ }^{2}$. Elle a renvoyé à la compréhension même de ce que doit être le syndicalisme dans une économie sociale de marché (die soziale Marktwirtscchaft ${ }^{3}$, à savoir un élément organisé du dialogue social, exerçant ses fonctions hors de l'entreprise, sans prétendre diriger ni exercer une quelconque autorité sur les conseils d'entreprise, même si en grande majorité ces derniers sont adhérents du syndicat. La stricte délimitation des pouvoirs impose aux conseils d'entreprise de ne pas supplanter les syndicats dans la représentation et la défense des intérêts salariaux dont les règles sont fixées au niveau de la branche, comme elle requière des syndicat de ne pas se

1. Dès le mois de février l'ancienne confédération FDGB a décidé de laisser libre les fédérations en matière d'adhésion. La mise en place de bureaux syndicaux de Land et de Kreise s'est étendue de juin 1990 à juin 1991. Au 31 décembre 1991, le DGB représentait $47 \%$ des salariés intégrés en 1989 au sein du FDGB, soit 4157000 (pour 9 millions d'adhérents en 1989). Ces 4 millions d'adhérents représentaient alors $60 \%$ des actifs. Bafoil (F.), L'Allemagne de l'est 1990-1993, destruction, crise, adaptation, thèse d'habilitation, IEP de Grenoble, 1994, 5e chapitre.

2. Sur la disparition du FDGB et le mouvement d'adhésion au DB, voir Lattard (A.), .Mouvement syndical et unification, Matériaux pour l'bistoire de notre temps, 23, 1991.

3. Le terme de Soziale Marktwirtscbaft caractérise en propre le système économique allemand dont la composante de la redistribution sociale est essentielle. Elle renvoie, en théorie, aux principes de l'ordo-libéralisme énoncé dans les années trente et repris après la guerre par les équipes du ministre de l'Économie Ehrard. Elle désigne, en pratique, les nombreuses procédures de réallocations des gains, selon le principe de la subsidiarité, fixant à chacun des partenaires publics et privés sa part dans la négociation collective, et à chacun des partenaires professionnels, sa fonction au sein de la négociation paritaire professionnelle. On peut parler alors de -partenariat social. (Sozialpartnerschaft). 
substituer aux acteurs de l'entreprise ${ }^{1}$. C'est cette distinction de la structure même du syndicalisme et de la cogestion que les salariés est-allemands n'ont pas compris initialement. L'autonomie dans la négociation des conventions collectives, en effet, ne se comprend pas seulement vis-à-vis de l'État mais aussi vis-à-vis de chaque partenaire. En raison, d'une part, de l'expérience faite du syndicalisme en RDA, courroie de transmission du parti dans l'entreprise et simple gestionnaire des affaires sociales ${ }^{2}$, et, d'autre part, de la composition des nouveaux conseils, élus dans la foulée de l'unification en 1990, il était attendu de ces derniers qu'ils remplissent le rôle de défenseurs des intérêts des salariés.

Largement discrédités, les anciens fonctionnaires syndicaux ont laissé la place dans la très grande majorité des cas aux militants du mouvement social ${ }^{3}$ qui ont conduit activement la urévolution* de 1989 et qui ont investi les conseils d'entreprise sur les lieux de travail. Pour nombre de ces militants, souvent recrutés parmi les techniciens et les ingénieurs, le directeur a été identifié sous les traits soit d'un collaborateur de l'ancien régime, soit d'un individu prêt, pour ses propres intérêts, à vendre l'entreprise à un investisseur étranger, dont la priorité sera de liquider l'emploi. Du fait de la forte participation des militants du mouvement social dans les conseils municipaux et dans les autres instances élues, et en raison de la détention par ces derniers de dossiers compromettants pour les anciennes élites dirigeantes, le conflit a eu tendance à être transposé très vite dans la sphère politique. Cependant, la composition des premiers conseils a évolué, les militants de la première heure ont été écartés, d'autres catégories ont accédé aux postes de responsabilité, notamment les employés de l'administration. Mais les relations entre organisations syndicales et conseils d'entreprise n'en ont pas moins connu de nombreuses frictions, les seconds passant souvent au delà des instances syndicales, comme lors de la création, en 1992, d'un collectif de conseils d'entreprises décidé à faire avancer seul ses revendications en matière d'emploi, et n'hésitant pas, cette même année, à en référer directement au Chancelier, par delà les procédures éprouvées et pour des prérogatives relevant directement des organisations syndicales.

La seconde difficulté a sans doute tenu à la composition des organisations sociales. À coup sûr, le problème du chômage s'est révélé être le problème le plus délicat ${ }^{4}$. Comment faire, en effet, lorsqu'une organisation syndicale se

1. La seule instance de représentation des intérêts des salariés dans l'entreprise employant plus de cinq salariés est le conseil d'entreprise (Betriebsrat), dont l'activité est régie par les lois de 1952 et 1972. C'est a lui qu'il revient de mettre en pratique les conventions collectives et qu'il incombe de signer avec l'employeur, au titre de la co-décision (Mitbestimmung), les documents concernant la durée du travail et les heures supplémentaires, la protection de la santé, la fixation des primes, les critères de sélection du personnel, le plan social de l'entreprise. Voir, Hassenteufel (P.), Systèmes de représentation et enjeux de pouvoir dans l'entreprise. Une comparaison France/RFA., La Reure de l'IRES, 3, 1990.

2. Bafoil (F.), Entreprises et syndicats en RDA. Une bistoire de l'émulation est-allemande, Paris, L'Harmattan, 1991.

3. Le mouvement social (die soziale Bewegung) désigne l'ensemble des groupes opposés à la dictature du SED, qu'ils aient existé sous le régime de RDA pour certains (comme le mouvement des droits de l'homme) ou qu'ils se soient constitué a l'automne 1989 comme le plus connu d'entre eux, le Neues Forum. Opposés, en règle générale, à l'action politique, ils ont avancé des revendications davantage éthiques que politiques, et ont disparu peu à peu de la scène politique moins d'un an après leur apparition. Rucht (D.), German Unification, Democratisation and the Role of Social Movements : A Missed Opportunity., WZB-Paper, FS III, 95-103.

4. Il convient ici de rappeler que les courbes du chômage et celles du chômage technique (Kurzarbeit) ont connu une croissance très rapide entre le mois de juillet 1990 ou elles étaient respectivement à 272017 et 656277 , le mois de décembre de la mềme année où elles ont atteint [suite de la note page suivante] 
compose essentiellement de futurs chômeurs ? Comme l'a affirmé le responsable de l'IG Metall de Leipzig, alorsque seulement une minorité de nos membres tire ses revenus directement de l'accord salarial, on doit chercher d'autres formes d'action organisationnelles* ${ }^{1}$. Les demandes exprimées par les femmes en matière d'emploi ont, tout autant, pris les organisations au dépourvu : elles qui ne constituent qu'un tiers des actifs ouest-allemands, alors qu'elles étaient, en RDA, à plus de $90 \%$ pour les $18-60$ ans, soit au travail, soit en formation ${ }^{2}$. À l'inverse, ce sont elles qui ont constitué plus de la majorité des chômeurs à l'Est (leur nombre excède les $60 \%$ sur la période) et qui se sont trouvées cependant le moins concernées par les mesures de formation, compte tenu de la nature des formations offertes. Enfin, ce sont elles qui ont réclamé des droits que la société ouest-allemande refuse à leurs homologues de l'Ouest, comme celui ayant trait à l'avortement, dont le principe était acquis depuis longtemps en RDA. Dans l'incapacité de répondre à ces demandes, les organisations sociales ont parfois laissé la place à des organisations locales, dont la plus importante est l'Association des chômeurs d'Allemagne (Arbeitslosenverband Deutscblands) ${ }^{3}$. Strictement limitée au territoire de l'ancienne RDA, en dépit de ses prétentions nationales, cette dernière n'en connaît pas moins des succès certains dans son effort d'intégration locale des exclus du travail, sous la forme de formation, d'emplois à durée déterminée, etc. ${ }^{4}$

Ce décalage entre les attentes provenant de populations adhérentes différentes s'est traduit par le heurt des revendications exprimées de part et d'autre, renvoyant à des expériences et des cultures radicalement opposées. Ainsi aux attentes en faveur du maintien de l'emploi envers et contre tout, au motif que les licenciements de masse affectent l'équilibre de régions entières, se sont opposées les revendications élaborées durant la décennie passée en Allemagne de l'Ouest, en matière de respect de l'écologie, d'abaissement du temps de travail, et d'introduction de nouvelles technologies. D'un côté, des garanties pour le travail à tout prix ; de l'autre, une demande de croissance des temps de loisir. La décision d'arrêter complètement des installations jugées polluantes s'est avérée un impératif à l'Ouest, mais à l'Est une atteinte aux équilibres humains. Or, ce décalage s'est doublé d'un second désajustement, cette fois lié aux niveaux d'intervention attendus de la part de l'organisation. Les salariés est-allemands ont fait état, en de nombreux cas, d'attentes d'une intervention en provenance des échelons supérieurs de l'organisation, considérée comme la seule réponse adéquate aux réalités du chômage de masse et de la privatisation, décidée ailleurs que localement. Ce

642200 et 1795300 , et enfin mars 1991, où elles se sont établies à 808389 d'un côté et 2001571 de l'autre. Par ailleurs de 1991 à 1994, si l'on cumule tous les types de sorties du marché du travail (formation, pré-retraites, chômage, chômage technique, mesures en ABM, etc.), on aboutit a un total oscillant entre $35 \%$ et $40 \%$ d'individus qui ont quitté le marché du travail. De 1990 à 1994 , l'emploi industriel est passé de 3,5 millions d'emplois a moins de 650000 .

1. Kletzin (J.), Politik und Organisation der IG Metall., in Schmid (J.), Löbbler F.), Tiemann (H.), dir., Probleme der Einbeit. Organisationstrukturen und Probleme von Parteien und Verbänden, Berichte aus den neuen Bundesländern, Marburg, Metropolis-Verlag, 1994.

2. Nicke (H.M.), Kühl (J.), Schenck (S.), Enverbsarbeit und Bescbäftigung im Umbrucb, Berlin, Akademie-Verlag, 1994.

3. Cette association a été fondée en 1990-1991 par un ancien sociologue, membre du PDS, ayant travaille antérieurement pour la FDGB. Bénéficiant des aides de l'Etat comme les mesures de création d'emploi (les ABM, équivalant grosso modo a nos anciens TUC), cette association dispose de relais dans les cinq Länder où elle concentre ses interventions.

4. Wolski Pengler (P.) -Arbeitslosenarbeit in den Neuen Bundesländern. Eine Bilanz nach drei Jahren Massenarbeitslosigkeit., Aus Politik und Zeitgescbicbte, 52, 1993. 
défaut volontiers qualifié à l'Ouest de abureaucratismen ou de réminiscence de comportements inadaptés, a fait l'objet depuis longtemps de discussions au sein des organisations des anciens Länder, dont l'un des objectifs repose sur l'action conjointe de syndicats unitaires, homogènes et décentralisés. Le renforcement de l'action syndicale n'a pas été posé à l'Ouest en termes de recentralisation de l'organisation. Or, comment expliquer aux adhérents estallemands du puissant syndicat du secteur public et des transports (ÖTV) que la structure très décentralisée du syndicat s'accompagne néanmoins d'un retrait hors de l'entreprise (là où il existait pourtant en RDA) et d'une intégration au sein des trois niveaux verticaux que sont le Kreis, le Bezirk et le bureau fédéral ? Plus encore, comment expliquer que la disparition de la représentation par entreprise implique également l'union de métiers très différents les uns des autres dans le syndicat, et par conséquent des compétences extrêmement élevées de la part des fonctionnaires, pour pouvoir assurer d'une même voix le respect de chacun d'entre eux ? ${ }^{1}$

Le processus d'unification a contribué, ainsi, à aggraver la situation du syndicalisme allemand déjà fragilisé dans la décennie quatre-vingt, au niveau de la composition des adhérents, caractérisée autant par une sousreprésentation des femmes et des jeunes, que par une montée du secteur des services à la faible activité collective, ou encore par une faible implantation dans de nombreuses PME. Il n'est pas jusqu'à une crise profonde qui n'ait affecté les secteurs traditionnels de la métallurgie, de la chimie et de la sidérurgie. En matière de relations avec les partis politiques, celles liées historiquement avec le SPD se sont distendues sans que s'affirment, pour autant, celles avec les Verts, dont une partie de la clientèle est composée des exclus du travail et des jeunes. Cependant, à l'Est, les syndicats se sont avérés la seule force constituée, en face des gouvernements régionaux, et à l'exclusion, pendant longtemps, des représentants patronaux.

\section{Les Unions d'employeurs}

Trois organisations représentent les employeurs à l'Ouest ${ }^{2}$. Or, à l'Est au début de l'unification, en raison de la composition même des employeurs de l'époque, elles se sont avérées inexistantes, à l'exception peut-être des chambres de commerce dont les cadres supérieurs ont été presqu'e intégralement composés de spécialistes de l'Ouest.

En 1990, les employeurs se divisent en deux catégories : on trouve d'abord les directeurs des grandes entreprises dont ne veulent absolument pas les organisations de l'Ouest, et qui créent une union sans importance, parce que vite sans appuis et dévalorisée en raison du passé de ses membres, "l'union

1. Hintz (C.), ÖTV in den neuen Ländern•, in Schmid (J.), Löbbler (F.), Tiemann (H.), dir., Probleme der Einbeit, op. cit.

2. La première confédération, chargée de défendre les intérêts économiques de ses membres, regroupe 34 unions d'employeurs et $94 \%$ des entreprises industrielles (BDI). Elle opère en plusieurs groupes spécialisés au niveau fédéral ainsi qu'au niveau des régions. La seconde (BDA) est exclusivement en charge des négociations tarifaires et de la politique sociale. Regroupant 46 branches (incluant 477 unions membres), BDA est marquée par une très forte concentration des branches, dont la conséquence se laisse lire au niveau de l'action collective, reposant sur une forte hétérogénéité de la structure des membres. $80 \%$ des entreprises sont adhérentes de BDA à l'Ouest. Troisième acteur relevant de la représentation professionnelle des employeurs : les chambres de commerce et d'industrie (DIHT). Henneberger (F.), Arbeitgeber- und Wirtschaftsverbände in den neuen Bundesländern : Konfliktlinien und Organisationsprobleme*, in Schmid (J.), Löbbler (F.), Tiemann (H.), dir., Probleme der Einbeit, op. cit. 
des employeurs de RDA. (Unternebmenverband der DDR). On trouve ensuite les patrons des entreprises reprivatisées, mais dont le nombre est initialement très faible. Une fois le processus de transformation de la propriété lancé, les différentes initiatives des employeurs ont été unifiées pour donner naissance à des unions régionales, dont l'un des traits marquants est de renouer avec les traditions des employeurs, datant d'avant 1939 et même pour certaines d'entre-elles d'avant la première guerre mondiale, à l'instar de l'union des employeurs de Saxe, fondée la même année que sa partenaire de Bavière, en 1902. Mais au moment où sont signés les premiers accords tarifaires, les organisations patronales ne sont pas représentatives, en raison de l'écrasante majorité des entreprises d'État et de la trop faible participation des PME. Plus encore, les employeurs habilités alors à signer sont ceux qui risquent le plus d'être éjectés de leur poste de directeur. Pour deux raisons, au moins, ils n'ont aucun intérêt personnel à signer des accords à la baisse. D'abord, parce que bientôt réduits à l'inactivité, ils ont intérêt à profiter de retraites élevées. Ensuite, parce que dans un contexte d'incertitude, il vaut mieux ne pas s'affronter aux syndicats. L'effet a été immédiat au niveau des accords signés en 1990 et en 1991, pénalisant gravement les employeurs durant toute la période. Ces derniers ont eu de surcroît à souffrir de ne pas pouvoir être entendus de la part de leurs homologues de l'Ouest lorsqu'ils ont dénoncé, comme les syndicats d'ailleurs, la profonde désindustrialisation de l'ancienne RDA. Faute de faire entendre leur voix tant au niveau des accords salariaux, qu'au niveau des organisations partenaires, seule la voie de l'exit leur est restée, que plusieurs d'entre eux ont emprunté, affaiblissant encore davantage un système de relations professionnelles déjà largement ébranlé.

Trois éléments ont ainsi concouru à entraver la bonne application du modèle des relations professionnelles ouest-allemand en Allemagne de l'Est, dans la première phase de l'unification : le temps, d'abord ; la nature et l'ampleur des problèmes, ensuite; l'absence de réponse pertinente, enfin. Triple déficit dont les conséquences ont été considérables, tant entre les deux communautés allemandes, qu'à l'intérieur de chacune d'elle. La crise des accords salariaux en est l'exemple type.

\section{Un transfert de régulation problématique}

Les accords salariaux ont constitué un des axes majeurs de l'activité syndicale en Allemagne de l'Est, compte tenu d'une part, des différences existant entre les deux Allemagne du point de vue de la productivité et des salaires ${ }^{1}$ et, d'autre part, de l'exigence inscrite dans le Traité d'union de 1990 concernant l'égalisation rapide des conditions de vie des deux parties de l'Allemagne unie. La crise des accords salariaux est-allemands a soulevé la question de la branche comme lieu pertinent de l'action collective. Ce faisant, en aiguisant un débat déjà largement abordé durant la décennie écoulée, elle a reposé le problème de l'homogénéité de la branche et de la performance de ses composantes individuelles, les entreprises, en les opposant. Tant que les performances économiques sont assurées et renforcées par un compromis social qui conduit, dans un cercle vertueux, l'ensemble des composantes

1. S'il s'est avéré très délicat, voire impossible, de préciser la valeur des taux de productivité par branche en 1990, compte tenu de l'incompatibilité des éléments pris en compte, en revanche, on peut situer le différentiel salarial entre les deux Allemagne à cette époque dans un écart de 1 à 5 . En 1990, le salaire moyen est-allemand a pu être fixé aux alentours de $35 \%$ du niveau de l'Ouest. En 1991, il n'était plus en moyenne que de $\mathbf{4 1} \%$. 
d'une branche à la production de standards sociaux élevés, voire comme dans le cas de la formation professionnelle à un partage des tâches entre entreprises performantes et celles qui le sont moins, chacun y gagne. La centralisation et l'homogénéité se renforcent mutuellement. À partir du moment où les profits réalisés ne sont plus en mesure de satisfaire les augmentations salariales attendues, la centralisation n'est plus nécessaire aux yeux des employeurs, l'homogénéité est un obstacle puisqu'elle impose une mesure erronée pour les entreprises qui ne peuvent s'y rapporter, et la décentralisation s'apparente à la dérégulation puisque le niveau pertinent devient l'entreprise et non plus la branche.

Faut-il pour autant suivre Birgit Mahnkopf lorsqu'elle affirme que les dérèglements liés à l'unification allemande ainsi que la situation déplorable des entreprises est-allemandes ont été une occasion saisie par les représentants du gouvernement pour s'attaquer à l'une des conquêtes les plus importantes de la démocratie sociale, notamment l'aautonomie tarifaire* ?1 Selon la sociologue allemande, l'abaissement au niveau de l'entreprise des négociations relevant antérieurement de la branche mérite d'être analysé comme un élément s'inscrivant dans la stratégie d'affaiblissement de la régulation sociale et de recomposition des rapports sociaux, en faveur des employeurs et de l'État. On serait tenté de suivre plus volontiers Horst Kern qui, tout en affirmant lui aussi le principe de la crise du rapport salarial et donc la crise de la régulation ouest-allemande, propose de la comprendre davantage comme une occasion offerte aux partenaires sociaux d'innover en matière de régulation sociale, tant au niveau du lieu de la négociation qu'à celui des attentes en matière sociale ou économique ${ }^{2}$. On reviendra plus bas sur cette exigence qui s'est imposée à l'Allemagne unie et qui a trouvé un commencement de réponse en 1995. Soulignons pour l'instant que la crise est-allemande, provoquée notamment par l'émergence de blocages dus à un transfert intégral des règles ouest-allemandes, a eu des effets en retour très importants contre le principe d'action centralisée. En d'autres termes, la procédure initiale de centralisation du transfert des règles s'est trouvée enrayée par les blocages qu'elle a elle-même contribué à produire.

\section{Les principes de la négociation paritaire allemande}

La négociation paritaire professionnelle traitant des relations propres aux branches industrielles est un élément décisif de l'équilibre allemand. Elle porte un nom : l'autonomie tarifaire (die Tarifautonomie), garantie par l'article 9.3 de la Loi fondamentale. Autonome, la négociation l'est dans la mesure où elle renvoie aux organisations professionnelles, patronat et syndicats, qui sont seules chargées de négocier entre eux les éléments des conventions collectives. Cela signifie que l'État n'est pas partie prenante du jeu, pas plus que l'entreprise, tenue à l'écart des négociations. Les conventions établissent, par branche, les règles en matière de durée du travail et de temps de repos, de qualification et de classification, et, bien entendu, en matière de

1. Mahnkopf (B.), The Impact of Unification on the German System of Industrial Relations,, PSI, 93-102, WZB-Paper.

2. Kern (H.), -Intelligente Regulierung*, Soziale Welt, 1, 1994. H. Kern est le directeur de l'Institut de recherche sociologique, le SOFI, de l'université de Göttingen. Il est l'auteur avec M. Schumann, de l'ouvrage, Industriearbeit und Arbeitsbeunsstsein, Surkhamp, 1970, ainsi que de l'ouvrage traduit en langue française sous le titre La fin de la division du travail, Paris, Editions de la MSH, 1988, qui a eu un très large écho. 
rémunération. En règle générale, les négociations s'effectuent au sein d'un Land défini comme pilote, en raison de la présence sur son territoire d'un grand nombre des entreprises concernées, et les résultats s'étendent à la branche entière. Si l'autonomie tarifaire illustre la capacité des acteurs sociaux à fixer le cadre de leurs stratégies, elle n'en est pas moins très contraignante pour ceux qui y sont soumis, dans la mesure où ils sont tenus, dans l'entreprise, d'en appliquer les contenus. Les syndicats disposent, certes, de la possibilité de faire grève, mais celle-ci ne peut être décidée que si plusieurs procédures ont été épuisées. Par ailleurs, des clauses dérogatoires (Offnungsklauseln) peuvent autoriser, sous conditions très strictes, les employeurs à introduire des éléments de flexibilité, à partir des résultats de leur entreprise. En fixant au niveau de la branche le contenu des conventions et en l'imposant aux entreprises qui y appartiennent, le système des relations professionnelles assure à la fois un haut degré d'homogénéité des branches et une faible marge de manœuvre à des acteurs étroitement contraints au sein du jeu institutionnel. La recherche du compromis exigé par la nécessité de signer les accords et de reproduire l'équilibre social, souligne, en effet, le manque de flexibilité du système, la faiblesse d'innovation des acteurs, et, à l'inverse, le risque attaché à sa transformation, le coût lié à la grève, la difficulté de renouveler les règles du jeu.

\section{La structure des salaires est-allemands}

Le premier round salarial a eu lieu avant même l'union monétaire, en mai et juin 1990, pour être validé à la date du 1er juillet 1990. Les augmentations pratiquées ont alors été considérables puisque si l'on prend en compte tous les éléments ajoutés (augmentations ainsi que primes de vacances, primes de sécurité, etc.), elles ont correspondu à un mois de salaire brut supplémentaire en moyenne. Le second round salarial a débuté à l'automne et a débouché sur l'accord-cadre de la métallurgie, fixant pour le processus d'égalisation, prévu à la fin 1994, une série d'étapes fonctionnant comme autant de paliers de rattrapages. Pour l'année 1991, les augmentations ont été de l'ordre de $60 \%$ et pour l'année suivante de $20 \%$. Si, en 1990 , le salaire moyen se situait à $35 \%$ du niveau de l'Ouest, en 1991 il n'était plus que de $47 \%$. Ces chiffres méritent cependant d'être rapportés aux autres augmentations qui ont affecté les coûts de la vie dans la période. La chute progressive des subventions et les augmentations des prix ont en réalité annulé partiellement les augmentations obtenues car pour une croissance des coûts de $20 \%$ en moyenne en 1991, celle des salaires nets moyens des ouvriers a été de $17 \%$ et celle des salaires nets moyens des employés de $26 \% 1$. Les gains ont été plus substantiels en 1992 dans la mesure où la croissance des coûts et des prix s'est tassée. Sur l'ensemble de la période de la mi-1990 à la fin 1992, la croissance nette des salaires a été de 40,9\% (55,3\% bruts) mais celle du pouvoir d'achat, compte tenu des diverses hausses, s'est établie à $12,6 \% 2$.

1. Au total, en $1991,16 \%$ des individus de l'Est gagnaient moins de 1000 DM par mois (pour $6 \%$ en RFA) et $26 \%$, plus de 3000 DM (pour $61 \%$ à l'Ouest). Si la croissance d'un revenu familial composé de trois personnes (ouvrier et employé) a cra de 1989 à 1991 de $160 \%$, en revanche, au cours de la même période, les coûts de la vie (chauffage, eau, électricité) ont connu une hausse de $350 \%$ et ceux de l'habillement de $110 \%$

2. Bispinck (R.), Sind die Löhne schuld ? Die Tarifpolitik in den neuen Ländern im Jahr 1992., WSI-Mitteilungen, 3, 1992. 
Plus encore, ces chiffres méritent d'être corrigés en prenant en compte les différences régionales, les différences sectorielles, les différences intrasectorielles, ainsi que les différences de composition des salaires. Les premières renvoient à la distinction capitale/province : les emplois ont davantage crû à Berlin qu'en province, et dans la capitale les taux d'égalisation ont été plus rapides, en règle générale, que dans les cinq nouveaux Länder. Les différences sectorielles, en second lieu, révèlent une grande amplitude entre les branches, car si, à la fin de 1992, la différence entre les branches de l'Est et celles de l'Ouest se situe entre $65 \%$ et $75 \%$, on note que le niveau moyen dans l'industrie se situe aux alentours de $60 \%$, et, dès la fin 1991, une branche comme le nettoyage connaissait un taux de $100 \%$ d'égalisation avec la branche correspondante à l'Ouest. Par ailleurs, tant en matière de durée hebdomadaire de travail que de durée des vacances, les différences Est-Ouest sont importantes : $42-45$ heures et $20-21$ jours de congés à l'Est pour 38,4 heures et $28-32$ jours à l'Ouest. Troisième type de différence, les différences intersectorielles qui opposent notamment la branche de l'habillement (904 DM en moyenne en 1991) au secteur le mieux payé à Berlin, les carreleurs (2180 DM en moyenne). Les différences de rémunération des ouvriers spécialisés dans les deux branches sont de 2,4 tandis que les plus grandes amplitudes à l'Ouest sont de 1,8. Dernière série de différences, celles qui touchent à la composition des éléments du salaire, en particulier, l'absence fréquente à l'Est de suppléments payés à l'Ouest, et lorsqu'ils sont versés à l'Est, leurs montants sont moins élevés. La comparaison des deux types de salaires versés dans la métallurgie pour le premier en Saxe, pour le second en Bavière est importante pour comprendre l'enjeu du conflit qui s'est déroulé au printemps 1993 dans ce secteur. Nominalement l'équivalence est de $82 \%$ entre les deux Länder. Si l'on prend en compte le fait que ni la prime de vacances, ni celle distribuée par la région ne sont attribuées à l'Est, et que de surcroît la durée annuelle de travail est plus longue à l'Est, on obtient une équivalence réelle de $63,9 \%{ }^{1}$. Ces différences ont poussé les syndicats de la métallurgie à se lancer dans la confrontation au printemps 1993, après que le patronat a lui-même rompu les négociations, arguant que les différentiels de productivité et de salaires demeuraient par trop élevés, entraînant ainsi l'impossibilité pour les entreprises d'assurer leurs charges.

\section{La crise de 1993}

L'harmonisation des conditions de vie des citoyens est- et ouest-allemands a été inscrite au titre du Traité d'union. Cela signifie que le législateur a prévu le rattrapage et l'égalisation des différences, et cela indépendamment parfois des données, notamment économiques, est-allemandes. On l'a vu avec les taux adoptés lors de l'union monétaire, décidés sans aucune prise en compte ni de la situation des entreprises ni du différentiel productivité/salaire ${ }^{2}$. Dès lors, les dysfonctionnements se sont multipliés. La productivité du travail et la rémunération de l'effort ont été largement disjoints : les taux de productivité ont accusé une chute, freinée seulement par les réductions d'emploi, alors que les salaires ne cessaient de connaître des hausses successives. Ce divorce, dénoncé sans discontinuer par les économistes au motif qu'il engendre des

1. En Saxe, la durée annuelle de travail est de 1792 heures, pour 1584 en Bavière.

2. Andreff (W.), -L'Allemagne de l'Est, modèle économique pour l'Europe de l'Est. De la voie royale au cul de sac., in Bafoil (F.), Samson (I.), dir., L'Allemagne en puissance, Paris, Editions Descartes, 1991. 
transferts monétaires toujours croissants, la fragilisation des entreprises, et finalement un grand nombre de faillites, a trouvé son point culminant lors du conflit qui a opposé les partenaires sociaux de la métallurgie au printemps 1993. Ce conflit prend sa source dans le refus par les représentants de l'organisation patronale de Saxe, puis des autres Länder est-allemands, de respecter la clause signée avec l'IG Metall, en 1991, de rattrapage des salaires Est-Ouest au printemps 1994 (soit une augmentation de $26 \%$ au 1er avril 1993). En quittant la table des négociations et en reprenant leur parole, les représentants des employeurs ont mis en question le type de négociation paritaire au fondement de l'équilibre social allemand. Les arguments avancés par chacune des parties ont avant tout été d'ordre politique, mais en renvoyant à chaque fois à des présupposés d'ordre économique et social.

L'argument politique des syndicats s'est appuyé sur l'exigence de citoyenneté posée par le Traité d'union, faisant obligation aux partenaires sociaux et politiques de tout mettre en œuvre pour y parvenir dans les plus brefs délais. Refuser cette égalisation, c'était risquer de voir reprendre les vagues d'émigrations d'Est vers l'Ouest, dont on se souvient qu'elles ont provoqué la demande d'unification monétaire. Le paradoxe aurait été ainsi que le phénomène migratoire, qui était un argument décisif pour la décision d'étendre le DM à la zone-Est, se retourne avec un effet tout aussi dévastateur ${ }^{1}$. L'argument économique en a découlé : il s'est agi de fixer la main-d'œuvre qualifiée et de l'empêcher de quitter le sol est-allemand, car cela n'aurait fait qu'amplifier la crise à l'Est pour laquelle des transferts financiers toujours croissants auraient été nécessaires. Les risques auraient été grands de voir ainsi les troubles sociaux se multiplier puisque l'on aurait vu apparaître des inégalités à l'intérieur de l'Allemagne de l'Est et plus encore entre les deux parties de l'Allemagne. C'est donc bien à l'aune des exigences politiques de l'Union qu'il fallait évaluer les risques économiques et non pas l'inverse. Les syndicats, selon cet argumentaire, se sont sentis d'autant plus les défenseurs de l'équilibre social allemand que les employeurs, à leurs yeux, ont cherché par tous les moyens à abaisser la négociation au niveau de l'entreprise, ce qui aurait eu pour effet de défavoriser les salariés les moins protégés et de menacer gravement l'unité tant dans les branches qu'au niveau national. C'était là, en effet, remettre en cause la soziale Manktwirtschaft comme système économique et social.

À cette accusation de vouloir casser l'ordre social, les représentants des employeurs ont rétorqué en reprochant aux syndicats de casser l'ordre politique car, à leurs yeux, ce sont les organisations sociales qui ont voulu sortir du cadre constitutionnel et abuser de leurs droits. L'article 9.3 de la Loi fondamentale a fait en effet obligation aux partenaires sociaux dans le cadre de la négociation paritaire de branche d'assurer et de soutenir les conditions économiques et de travail. En ne voulant pas se rendre aux arguments économiques selon lesquels les taux de productivité ont été largement inférieurs aux taux de croissance des salaires, les syndicats ont mis en péril non seulement les équilibres économiques mais également les équilibres sociaux. Outre que ce sont près de 70000 emplois qui ont été de la sorte directement menacés, selon eux, c'est l'ensemble du tissu social qui a été atteint avec cette croissance du chômage que les syndicats ont contribué à

1. Le mot d'ordre scandé par les manifestants de l'hiver 1989 était bien : Si le DM ne vient pas à nous, c'est nous qui irons au DM. 
renforcer. S'affirmant toujours attachés à la négociation paritaire de branche et soucieux de l'égalisation à terme des conditions salariales Est-Ouest, les représentants patronaux ont réclamé l'adaptation des conventions aux conditions locales des unités de travail.

Ce conflit a débouché sur la décision de faire grève dans le Land de Saxe. L'enjeu s'est révélé considérable, non seulement du strict point de vue de la négociation paritaire, mais peut-être plus encore du point de vue de la mobilisation. Il s'est agi là, en effet, de la première grève conduite par les syndicats sur le sol saxon depuis 1933. De son suivi dépendait, pour partie, la légitimité de l'IG Metall aux yeux de nombreux observateurs. Massivement soutenue, la grève a menacé à la fin du mois de mars de s'étendre à l'ensemble de la branche allemande. Or, la crise a trouvé son épilogue dans le compromis signé par les deux parties. Trois éléments méritent d'être retenus : la décision de reporter l'égalisation au 1er juillet 1996 ; la limitation du taux de croissance salariale à $9 \%$ (et non à $26 \%$ ); la possible renégociation par entreprise des contenus de la négociation, si les conditions économiques imposent une révision par le bas des engagements pris. Des clauses d'exception (Harteklauseln) ont en effet été introduites permettant, sous conditions expresses et dans des limites temporelles ne pouvant excéder 1996, certaines dérogations en matière de fixation des contenu de la convention. Cette clause a été évaluée par les milieux patronaux comme aune innovation positive en matière de politique tarifaire 1 . Cependant, leurs propositions d'inviter les conseils d'entreprise à la table des négociations n'ont pas été retenues et pas davantage celles visant à instaurer des minima par entreprise. Finalement l'esprit de compromis l'a emporté, le représentant du patronat cherchant a minimiser les différences d'appréciation : "Sur tous ces principes, a-t-il affirmé, nous n'avons pas finalement de positions radicalement dissonantes avec IG Metall; nous avons seulement des problèmes de formulation: ${ }^{2}$. Si IG Metall a montré qu'il était capable de mobiliser ses adhérents est-allemands, dont le degré de combativité a surpris, il a dû cependant céder sur une de ses exigences majeures, celle qui concerne le maintien de la branche comme lieu de la négociation et le non abaissement de la négociation au niveau de l'entreprise.

Comment expliquer ce qui a pu apparaître comme une reculade, alors que la mobilisation semblait jouer en faveur de la satisfaction des revendications syndicales? Pour comprendre l'issue donné à ce conflit, sans doute est-il nécessaire de rappeler le rôle essentiel rempli par la Treuhand ${ }^{3}$. L'essentiel des efforts de l'Agence centrale de privatisation a en effet visé à limiter les coûts. Ainsi, pour contraindre le syndicat allemand à accepter les exigences patronales, la Treuhand l'a menacé de laisser jouer pleinement les règles du marché, la conduisant inéluctablement à abandonner les entreprises insolvables, à se retirer des sociétés d'emploi, et plus généralement à liquider toutes les solutions élaborées depuis 1991 avec les syndicats pour réduire les effets de la crise de l'emploi. Or, ces coopérations ont constitué un des fondements majeurs de la légitimité syndicale durant la période,

1. Handelsblatt, 22 juin 1993.

2. Handelsblatt, 17 mai 1993.

3. Czada (R.), -Das scheue Reh und die Kröte. Investition und Beschäftigung im Kalkül der Treuhandanstalt, in Heinelt (H.), Bosch (H.), Reissert (B.), dir., Arbeitsmarktpolitik nacb der Vereinigung, Berlin, Sigma, 1994. 
particulièrement celle de IG Metall dont l'action en Saxe a été décisive pour le redéploiement de ce Land.

\section{Du transfert de régulation à la redéfinition du compromis socio-économique}

L'originalité de l'action syndicale en Allemagne de l'Est, durant la période de 1990-1995, a sans doute été de parvenir à dépasser le clivage qui fonctionne à l'Ouest entre politique sociale réservée partiellement aux syndicats, et politique industrielle, chasse gardée des responsables économiques et politiques. En de nombreuses circonstances, ils ont su nouer le dialogue avec la Treuhand, d'un côté, avec les partenaires politiques des Länder et des communes de l'autre, avec les employeurs dans un troisième cas.

Une innovation sociale majeure a été mise au point par les autorités publiques et par les syndicats, dans un domaine qui a constitué un des enjeux de la période, celui de l'emploi. Cela a concerné initialement les :mesures de création d'emploi. (Arbettsbeschaffungsmassnabmen, ABM) puis les sociétés d'emploi (Beschaftigungsgesellschaften), dont plusieurs aménagements locaux ont diversifié les formes, soit en raison des partenaires fondateurs, soit en raison des catégories visées. Ce faisant, les syndicats ont su mobiliser leurs adhérents sur l'une des revendications principales de la période, la sauvegarde de l'emploi. Renouant les fils de la négociation centralisée avec les partenaires publics centraux (notamment la Treuhand), et décentralisée avec les politiques régionales (le gouvernement du Land), ils ont su profiter de la faiblesse des partenaires employeurs, pour se poser en interlocuteurs privilégiés du dialogue social et mettre au point des compromis avantageux. Du strict point de vue des contenus de la négociation conduite avec les partenaires centraux et avec les partenaires régionaux, les syndicats, et notamment IG Metall, se sont largement singularisés par rapport à leurs homologues de l'Ouest, tenus généralement à l'écart de la mise au point de tels dossiers. Plus encore, ils ont su résoudre de manière originale la double tension de la mobilisation et de la participation au jeu institutionnel d'une part, de la centralisation des procédures et de la décentralisation des lieux de l'action, d'autre part.

C'est au terme d'une longue controverse avec les syndicats que la Treuhand a finalement accepté, en juillet 1991, de financer en partie le montage des sociétés d'emploi. Son refus initial de s'associer aux partenaires locaux et régionaux de ces entreprises était lié au fait qu'ayant déjà en charge la gestion du patrimoine est-allemand à des fins de vente ou de liquidation, il ne lui revenait pas, selon elle, de créer d'autres entreprises. Il n'était pas question, en effet, de créer des entreprises à son actif au moment où il lui était imparti de se défaire du stock mis à sa disposition. Quant aux autres acteurs - Land et syndicats, mais également conseils d'entreprises concernées - ils s'en sont tenus à la nécessité, selon eux, de ne pas exclure des centaines de milliers d'individus mais de contribuer, par leur retour à l'emploi, d'une manière ou d'une autre, au redéploiement des régions en crise. Une fois encore, l'opposition est passée entre logique économique d'un côté et logique sociale de l'autre'. Dès le 18 septembre 1990, l'organisation patronale (BDA) et la

1. Bafoil (P.), -La Treuhandanstalt, entre logique économique et logique sociale., Poutoirs locaux, 1992. 
confédération syndicale (DGB), toutes deux de l'Ouest, ont signé un texte intitulé *Pour plus d'emplois en RDA*, préconisant la création de sociétés de qualifications cliées aux problèmes sociaux du changement structurel en cours et susceptible ensuite, de recomposer les espaces d'entreprises. En mars 1991, la crise de l'emploi est devenue trop importante pour que la Treuhand puisse rester plus longtemps de côté, et le Chancelier lui-même a enjoint à l'Agence de privatisation de participer avec les autres acteurs publics et sociaux à l'élaboration de solutions collectives. Au début du mois de juillet 1991, l'office de privatisation a accepté de participer avec les syndicats, les Länder et les patrons au montage de ces sociétés à concurrence de $10 \%$. Leur objectif est d'employer les bénéficiaires des mesures d'emploi (l'équivalent de nos TUC) dont le chiffre s'est élevé à 400000 à la fin 1991 .

Les sociétés d'emploi, de qualification ou de restructuration (Beschäftigungsund Qualifizterungsgesellschaf, Strukturentwicklungt), chargées, dans plusieurs cas, d'intégrer les ABM, sont devenues souvent les premiers employeurs de leur région. Fonctionnant comme des intermédiaires au sein d'espaces en recomposition, elles ont joué un rôle important dans l'espace économique est-allemand puisqu'il leur est revenu d'assurer travail et formation à bon nombre d'individus licenciés. L'objectif poursuivi à l'Ouest avec de telles sociétés était d'assurer une solution transitoire, le temps de permettre aux salariés concernés de se requalifier. Le concept de Warteschleifenmodell a ainsi été mis au point par les Konzern de l'acier à l'Ouest pour indiquer la finalité de reconversion et de réemploi. Grâce à la combinaison de plusieurs moyens financiers, les relations de travail se sont trouvées prorogées, sous une forme ou sous une autre. L'objectif à long terme a été de permettre aux travailleurs de s'installer à leur compte, requalifiés et réorientés dans les circuits de l'échange, en se posant comme les partenaires de leurs anciens employeurs dans le cas où ces derniers fonctionnent encore. Il s'est donc agi de revitaliser le tissu de la sous-traitance défaillant ${ }^{1}$.

Or ces Beschaftigungsgesellschaft n'ont pas cessé d'être au centre des critiques depuis leur création, La première de ces critiques a concerné les coûts : par exemple, la société de Bitterfeld - créée pour répondre au désastre de l'industrie de la chimie dans cette région - a englouti des sommes considérables (610 millions de DM). La seconde critique a dénoncé l'aspect contre-productif, voire anti-économique de ces sociétés : dans la mesure où elles ont eu pour segment du marché celui-là même qu'essayaient de conquérir a grand peine les petites entreprises du bâtiment ou des services, elles ont été dénoncées comme des concurrentes déloyales. Pratiquant des prix de dumping et jouissant de moyens financiers plus importants que leurs concurrents de la petite industrie, elles ont condamné peu ou prou ces derniers. Plusieurs résultats d'enquêtes sont venus infirmer ces critiques. Mais surtout ces dernières ont négligé un aspect fondamental de ces sociétés, celui de la constitution, grâce à elles, du second marché du travail. Outre le fait qu'elles ont permis $\grave{a}$ plusieurs dizaines de milliers de travailleurs de valoriser leurs qualifications et de demeurer motivés dans la recherche d'un autre emploi, ces sociétés ont largement favorisé la politique structurelle des

1. Une distinction doit être faite ici entre les très grandes sociétés d'emploi qui ont regroupé, à l'instar de celle de Bitterfeld, plusieurs milliers d'individus et celles, plus petites, dans des régions sans aucun poids, ni social ni économique. Alors que les premières ont disposé de capitaux et de soutiens importants au niveau des municipalités et du Land, les secondes n'ont profité que du soutien local. Elles ont d'ailleurs pour leur très grande majorité disparu a la fin de 1993. 
régions et des communes. Ces dernières soucieuses d'attirer les investisseurs dans des surfaces aménagées ont, en effet, largement fait appel à leurs services, orientés de plus en plus au fil du temps vers des travaux d'aménagement communal, de destruction des anciens bâtiments, de dépollution, etc.

En 1993, en raison des coûts très élevés entraînés par l'attribution des ABM et plus généralement des mesures de traitement social de chômage ${ }^{1}$ ainsi que des critiques adressées à ces mesures sociales, les autorités de Bonn ont décidé de redéfinir leurs orientations. De ce fait, les règlements qui précisaient antérieurement l'activité des sociétés d'emploi ou de restructuration ont été refondus et ont donné lieu à l'élaboration d'un projet - dit paragraphe $249 \mathrm{H}$ du code de soutien au travail Arbeitsforderungsgesetzt. Ce projet a précisé que les mesures de traitement social du chômage - ABM - seraient prioritairement accordées à des fins de protection et de retraitement de l'environnement ${ }^{2}$, pour trois branches exclusivement : celle des mines et des aciéries, de la chimie et de la métallurgie. À l'issue des différents contrats signés entre la Treuhand et les Länder concernés, ainsi qu'entre les bureaux locaux du travail et les partenaires syndicaux, 230 projets ont été retenus à la fin 1993, couvrant ainsi 35000 salariés. De l'accord signé en mars 1993, on retiendra que certains salariés sont dorénavant couverts, du point de vue des conventions collectives, par des accords spéciaux valant pour l'entreprise dans laquelle ils ont été employés et non par la convention de branche dont ils relevaient antérieurement. En d'autres termes, le salarié pouvant être affecté à d'autres tâches que celles que ses propres qualifications le destinent, n'est plus couvert par une convention de branche. Dans la mesure où sa nouvelle affectation a implique pour lui un déclassement, il ne peut plus se prévaloir de sa qualification antérieure pour son salaire mais bien de la seule activité accomplie dans la nouvelle entreprise.

Considérés sous l'angle des relations professionnelles, ces accords ont témoigné d'une profonde ambivalence. D'un côté, les entreprises ont été le théâtre de la mise en place de phénomènes de dérégulation qui ont affecté directement les salariés, puisque ces deniers n'ont plus été couverts par des accords de branche mais par des accords d'entreprise. De l'autre, la signature de ces accords a témoigné de la flexibilité des syndicats et de leur capacité à répondre de manière innovante à des situations de crise profonde. Le maintien de l'emploi est devenu un axe prioritaire des revendications, avant même celui de la garantie de salaire. Dès lors, l'instauration de plusieurs paliers dans le système des accords collectifs de branche a été posée comme la condition de survie du système des relations professionnelles tout entier. Créant certes une différenciation au sein même des branches et par conséquent une participation inégale à l'emploi, ces aménagements de la règle centrale n'en ont pas moins été considérés comme un moindre mal,

1. Le montant des dépenses financières au titre des mesures du traitement social du chômage s'est élevé en Allemagne de l'Est en 1991 et 1992 a 29,88 milliards de DM et 43,01 milliards de DM, tandis que celles réalisées a ces deux mêmes dates en Allemagne de l'Ouest se sont élevées à 42,05 milliards de DM et 42,21 milliards de DM respectivement.

2. Le retraitement de l'environnement a été mis au premier plan en raison des pollutions en grand nombre et de toutes sortes qui ont affecté l'air, l'eau ou encore la terre. Par ailleurs, la déstructuration des combinats et la mise en faillite de nombreuses entreprises a laissé inutilisé un grand nombre de bâtiments qu'il a fallu raser, afin de profiter au mieux des surfaces ainsi dégagées. 
dans l'attente d'une recomposition unifiée des branches. À cet égard, nul doute que ce qui s'est joué en Allemagne de l'Est en matière de flexibilité a préfiguré les développements qui ont vu le jour en Allemagne de l'Ouest, à compter de 1994. En cela, la crise est-allemande aura été source d'apprentissage pour les organisations et pour les acteurs de l'Ouest.

Cela vaut également pour tout ce qui a concerné la politique industrielle initiée par les Länder et par la Treuhand, à laquelle les syndicats ont été très étroitement associés. À chaque fois la décision de maintenir l'emploi a été prise à la suite d'un marchandage qui a vu la Treuhand accepter de soutenir des entreprises insolvables, à condition, d'une part que les autorités publiques régionales y affectent des fonds, normalement réservés à des entreprises privées, et d'autre part que les syndicats acceptent non seulement le principe de flexibilité des emplois, mais également celui de la limitation des revendications salariales. De très nombreuses critiques se sont élevées parmi les rangs des experts économistes et des employeurs pour dénoncer cette valorisation de l'emploi, aux dépends de la situation réelle des entreprises ou des dépenses publiques. Le plan le plus connu à cet égard a été le programme saxon Atlas, destiné à sauvegarder une centaine d'entreprises n'ayant pas trouvé de repreneurs sur le marché, et pourtant considérées par les pouvoirs publics comme restructurables, en raison de leur importance dans leurs bassins locaux respectifs. Lorsque le plan a été mis sur pied, un an plus tard, IG Metall en a été partie prenante ${ }^{1}$. Des plans similaires ont vu par la suite le jour ${ }^{2}$, davantage centrés sur une branche industrielle, comme celle des chantiers navals, avec le plan Mecklenbourgeois dit "Anker* ou le plan chimie en Saxe Anhalt ${ }^{3}$, ou sur une entreprise-phare d'un Land, comme l'aciérie EkoStahl de la ville brandebourgeoise d'Eisenhüttenstadt ${ }^{4}$.

\section{Les effets en retour de l'unification Vers la transformation du "modèle allemand"?}

La plus grande crainte des syndicats dans la période postérieure à l'unification a sans doute été celle de voir se constituer à l'Est une zone à faible coût salarial entraînant de nombreuses dérégulations, dont les effets en retour pour l'organisation toute entière se seraient révélés non maîtrisables. Mais, ̀̀ l'inverse, la poursuite sans discontinuer d'une stratégie de croissance salariale, telle qu'elle a pu être mise en pratique à l'Ouest durant les décennies passées, s'est révélée grosse de multiples conflits au contact de la réalité issue de l'unification allemande et de la récession apparue en 1993. En ce sens, l'extension à l'Est du modèle des stratégies syndicales élaborées à l'Ouest, et, plus largement encore, celui du fonctionnement du système des relations professionnelles, a témoigné de profondes insuffisances et requis sa transformation. En cela, la crise est-allemande, même si elle n'a pas été à

1. Nolttke (D.), Industriepolitik in Ostdeutschland am Beispiel des Bundeslandes Sachsen., Aus Politik und Zeitgescbicbte, 29 avril 1994 ; Menant (A.), -La politique industrielle en Saxe, le Programme Atlas., Les cabiers de l'Observatoire de Berlin, 23 septembre 1993 ; Giraud (O.), -Relations industrielles et politiques publiques dans le Land de Saxe : le cas de la politique industrielle., Les cabiers de l'Observatoire de Berlin, 30 juin 1994.

2. Bafoil (F.), L'Allemagne de l'est 1990-1993, destruction, crise, adaptation, op. cit., chap. 7.

3. Kern (H.), Sabel (C.), -Die Treuhandanstalt : Experiementierfeld zur Entwicklung neuer Unternehmensformen., Das Unmöglicbe Wagen, op. cit.

4. Bafoil (F.), Gastou (A.), Giraud (O.), Monographie de l'entreprise E-, Les cabiers de l'Observatoire de Berlin, 17 octobre 1992, ainsi que Gastou (A.), Une aciérie du Brandebourg. Une stratégie de développement., Allemagne d'Aujourd'bui, 121, 1992. 
l'origine de celle qui a vu le jour en Allemagne de l'Ouest à compter de 1993, n'en a pas moins produit des effets sur les partenaires de la négociation en Allemagne de l'Est et de l'Ouest. Les dérégulations qui ont vu le jour à l'Ouest ont trouvé dans les transformations est-allemandes un premier champ d'expérimentation.

\section{La double contrainte}

En 1993, deux contraintes ont pesé massivement sur l'action syndicale et ont forcé, à terme, les organisations sociales à modifier leurs stratégies. La première, renvoie à la situation financière des communes et des offices publics à l'Est. Le Pacte de solidarité signé en 1993 témoigne de la capacité de l'ensemble des acteurs, et notamment de ceux de l'Ouest, à modifier les règles du jeu, sans en altérer le sens, mais en modifiant la place des joueurs et les charges pesant sur eux ${ }^{1}$. En devenant intégralement payeurs, les Länder de l'Ouest acceptent que leurs partenaires de l'Est soient pour longtemps les seuls bénéficiaires du système de péréquation financière horizontale, tandis que le Bund accepte de son côté l'alourdissement de ses charges. Pour les acteurs syndicaux, qui ne sont pas directement parties prenantes du Pacte, la leçon est claire. L'heure n'est plus à la poursuite d'accords salariaux tous azimuts. L'emploi s'impose comme la priorité, au risque, et de nombreux syndicats l'acceptent, d'abaisser les accords au niveau de l'entreprise. C'est le sens de l'issue donnée au conflit en Saxe au printemps 1993. Mieux vaut, en substance, une dérégulation contrôlée de certains accords tarifaires qu'une crise généralisée de l'emploi. Ainsi, face à une crise sans précédent de l'emploi, les solutions pragmatiques l'emportent et, avec elles, la capacité des syndicats à accompagner au mieux les inéluctables restructurations économiques. Cette position rejoint celle qui, venant de l'Ouest, préconise qu'à côté des mesures de partage du travail, de régionalisation et de décentralisation des décisions, soient avancée l'idée d'une dérégulation partielle, couplée à des efforts de formation afin de répondre aux exigences de diversification des produits. On assiste ainsi, à la lecture des différents programme syndicaux ${ }^{2}$, $a$ la prise en compte de ala fin des idéologies politiques" et à celle des revendications "quantitativistes" centrées sur les

\footnotetext{
1. Dans le cadre de la redistribution des sommes tirées de l'impôt au titre de la péréquation qui se joue d'une part entre le Bund et les Länder et d'autre part entre les Länder entre eux, une hiérarchie s'est imposée en Allemagne de l'Ouest entre Länder payeurs (Hambourg, Bavière, Bade Würtenberg, par exemple) et Länder receveurs (la Sarre ou encore Brême). L'innovation majeure du Pacte de solidarité a été de reporter la date fixée dans le Traité à 1995, articulant l'égalisation en matière de participation de la part des Länder de l'Est à l'effort commun. Ce faisant, le Pacte a modifié radicalement l'ordre entre les Länder, en rangeant tous les Länder de l'Ouest au titre des contributeurs, et tous les Länder de l'Est au titre de bénéficiaires. De surcroît, le Bund a décidé d'accroître sa contribution de reversement. En ce sens, le Pacte a participé dú renouvellement des bases de la solidarité allemande. Sur ce point, voir Handelsblatt, 17 mars 1993, Deutschland Archiv, avril 1993, p. 397, Bafoil (F.), L'Allemagne de l'est 1990-1993, destruction, crise, adaptation, op. cit., chapitre 9, Benz (A.), Seibel (W.), Mäding (H.), Verwaltungsreform und Verwaltungspolitik im Prozess der deutscben Vereinigung, Baden-Baden, Nomos Verlag, 1992. Pour une compréhension du système de la péréquation financière et de la réforme administrative qui lui est liée, on lira en français, Hoffman-Martinot (V.), Finances et pouvoir local. L'expérience allemande, Paris, PUP, 1987.

2. Lire sur ce point, Hoffmann (J.), Hoffmann (R.), Lange (D.), Mückenberger (U.), -Herausforderungen der Gewerkschaftspolitik nach dem Ende des Ost-West Konflikts-, Gewerkscbaftliche Monatsbefte, 5, 1993 ; Offermann (V.), -Krise der Gewerkschaften ?., Arbeitsund Sozialpolitik, 3-4, 1993 ; Jeschke (D.), .Zur Tariflandschaft in den neuen Ländern-, Gewerkscbaftlicbe Bildungspolitik, 7-8, 1993 ; Brücker (H.), .Politikversagen oder Marktversagen., Frankfürter Hefte, 6, 1993 ; Kuda (R.), -Antikrisenstrategien, aus gewerkschaftlichen Sicht., Frankfiuter Hefte, 6, 1993.
} 
augmentations de salaires. Le concept de stéponse flexible- de la part des syndicats s'impose face à la politique de dérégulation avancée par les milieux gouvernementaux et patronaux ${ }^{1}$.

La seconde contrainte concomitante tient a la récession, qui a frappé les autres économies occidentales un an auparavant, et qui affecte désormais l'économie allemande. Dopées jusque là par l'ouverture du marché estallemand et par les subventions de toutes sortes, les entreprises ouestallemandes ne l'ont pas ressentie directement. À partir de 1993, ses effets se conjuguent à ceux de l'unification allemande, et s'il est sans doute erroné d'imputer les coûts de la récession à la marche de l'unification allemande, il n'est pas moins certain que les deux cumulés révèlent au grand jour les faiblesses de l'économie allemande. Au titre de ces faiblesses, on doit compter la perte de nombreux marchés extérieurs, la concentration des efforts, dans les années quatre-vingt, sur des "niches» économiques désormais soumises à une concurrence effrénée, un certain recul de l'industrie allemande dans des secteurs de pointe de la haute technologie, et finalement l'assoupissement d'un bon nombre d'exportateurs sur les vertus supposées indéfinies du modèle made in Germany, dont les ingrédients inaltérables seraient la discipline et l'âpreté au travail, la qualité, la solidité des produits ${ }^{2}$. Or, ces vertus, les concurrents du Sud-Est asiatique, notamment, se font fort de les renouveler dans des termes originaux, qui ont nom toyotisme, Kaizen, et autres formes d'organisation du travail fondées sur une rapidité d'innovation et de rotation des produits, ainsi que sur une vision du management qui fait défaut à l'industrie allemande. Mais surtout, au titre des faiblesses, plusieurs partenaires politiques (avec les Libéraux du FDP) et économiques ne cessent de mettre en cause les coûts salariaux, les plus élevés au monde, et corrélativement l'insuffisante durée du travail et d'utilisation des machines.

De plus en plus souvent, les syndicats sont accusés de mettre en péril l'industrie allemande par leurs demandes de réduction du temps de travail. Mais les tensions ne jouent pas seulement sur le front du travail avec les employeurs. Dans l'organisation elle-même, les conflits s'accumulent. D'abord, en raison de la perte importante d'adhérents, conduisant notamment à des réductions sensibles de revenus financiers ${ }^{3}$. Sous l'effet de la crise, ensuite, les organisations sociales ouest-allemandes se trouvent sous la pression de nombreux militants de base agissant dans les conseils d'entreprise, décidés a laisser tout simplement tomber les "partenaires" de l'Est, devenus de dangereux concurrents sur des marchés trop étroits. Ainsi en va-t-il d'investissements mis en sourdine parce que leur mise en ouvre aurait pu fournir l'occasion d'expérimenter des formes d'organisations

\footnotetext{
1. Windolf (P.), -Vom Korporatismus zur Deregulierung. Thesen zum Strukturwandel der gewerkschaften*, Joumal für Sozialforscbung, 4, 1989.

2. Sur la crise du modèle de management ouest-allemand, mais également sur celle des différents modèles d'origine asiatique appliqués à la réalité allemande, on lira en français : *Regards sur l'économie allemande., Bulletins du CIRAC, 23, 1995, ainsi que Roth (S.), Les répercussions des nouveaux concepts de production sur les relations sociales : du conflit au consensus ? L'exemple de l'industrie automobile allemande., Info, 37, 1995.

3. Le DGB a gagné de très nombreux adhérents avec l'unification puisque, comme on l'a vu, le taux de syndicalisation s'est révélé plus élevé qu'à l'Ouest, de l'ordre de $48 \%$ à l'Est contre 32 à l'Ouest en 1991. Toutefois, les pertes entre 1991 et 1994 se sont élevées à 2 millions. Passé de 7,9 millions d'adhérents en 1990 à 11,8 millions en 1991, ce chiffre s'est abaissé à 9,7 millions en 1994 . C'est en Allemagne de l'Est en 1992-1993 que les pertes ont été les plus lourdes.
} 
préjudiciables à celles en vigueur à l'Ouest ${ }^{1}$. Le même scénario se répète lorsqu'à l'Est une entreprise renommée pour ses produits, et dont les acheteurs se trouvent à l'Ouest, met en péril les concurrents de l'Ouest et, finalement, est mise hors course en raison des longues traditions nouées avec le donneur d'ordre ${ }^{2}$. La situation des organisations d'employeurs n'est pas meilleure. Refusant les conclusions des accords que signent leurs organisations professionnelles, de nombreux entrepreneurs se décident à ne plus y appartenir ${ }^{3}$. L'heure semble ainsi a une profonde refonte des comportements, impliquant une redéfinition des statuts professionnels, en matière de flexibilité du travail, de salaires, de qualification, qui constituent le cœur de la négociation collective. Pour y parvenir, certains n'hésitent pas à réclamer pour les syndicats un plus grand contrôle économique, en échange d'une prise en compte plus nette, de leur part, des contraintes liées au changement de contexte mondial. Gestion et contrôle doivent désormais davantage s'interpénétrer pour mieux contrôler l'innovation.

Prenant appui sur les exemples de l'Est, le sociologue Horst Kern avance l'importance de l'innovation dont font preuve les syndicats à l'Est et réclame pour les partenaires sociaux de l'Ouest une meilleure prise en compte des contraintes pesant d'une part sur l'entreprise, d'autre part sur la régulation sociale. Plaidant pour une ségulation intelligente* au cœur de la modernisation, il réclame pour les syndicats le statut de "co-managers", à charge pour eux de ne pas prendre en point de mire de leur action le seul axe des revendications salariales, mais de s'associer plus étroitement aux destinées économiques de l'entreprise. À l'inverse, cela implique pour les employeurs, de ne pas s'en tenir aux simples réductions d'emploi pour réaliser la rationalisation de l'appareil productif́. Poursuivant dans cette direction et prenant acte de l'impossibilité de continuer dans la voie de la recherche d'un compromis salarial au seul niveau de la branche, le viceprésident de l'IG Metall, quant à lui, préconise, dans le cas où la négociation ne peut que s'abaisser au niveau de l'entreprise, de renforcer expressément la "cogestion": :Si la cogestion au niveau de l'unité de travail et de l'entreprise est complétée par la cogestion au niveau du poste de travail, alors ce sera plus vivable et d'autant plus efficace. Sans aucun doute, cela réclame un changement de pensée de la part des conseils d'entreprise. 5 . Au titre de ces changements le responsable syndical cite l'apprentissage de la délégation, celui de la discussion approfondie, de l'organisation et de la protection des

\footnotetext{
1. C'est le cas des halles de Mosel qui ont été construites pour accueillir les chaînes de montages d'automobiles de l'usine Volkswagen et dont il était prévu a l'origine que ce site constituerait un -laboratoire social. pour la firme. Sous la pression des syndicats de l'Ouest, l'initiative a été freinée et les investissements réduits. Les innovations sociales ont été reportées, les salariés de l'Ouest craignant qu'elle ne fournissent l'occasion aux employeurs d'expérimenter des formes d'organisation qui leur seraient préjudiciables.

2. Bafoil (F.), Umformtechnik-, Les cabiers de l'Observatoire de Berlin, 32, 1994, et .Les apprentissages de la transition dans l'entreprise est-allemande, a paraître dans Sociologie du traevail, 2, 1996.

3. En 1994, ces départs ont été évalués à environ $20 \%$. Ce phénomène a également été repéré à l'Ouest, où il a différencié largement les patrons des PMI de ceux des grandes entreprises. Afin de se concilier les suffrages des premiers, les représentants patronaux ont adopté une attitude dure dans les négociations avec les syndicats, alors que la signature des conventions était dans la majeure partie des cas favorable aux grandes entreprises. Voir Hege (A.), -Allemagne, un compromis salarial presque ordinaire, IRES. Cbronique Internationale, 33, 1995.

4. Kern (H.), -Drei Thesen zur Gewerkschafts- und Gesellschaftspoltik", Interessenvertretung, Organisationsentwicklung und gesellscbaftsreform, Gewerkschafts - und Gesellschaftspolitisches Forum der IG Metall, 15-16 juin 1995.
}

5. Riester (W.) -Perspektiven zukunfiger Tarifpolitik., Jabrbucb für Arbeit + Tecbnik, 1995. 
salariés. En d'autres termes, ce qui semble se profiler derrière ce nouvel arrangement, c'est une remise à plat des conditions de travail et de l'organisation du travail pour des partenaires ayant évolué dans leur prise en charge des destinées de l'entreprise. Au changement de contenu et de niveau de la négociation doit correspondre un changement de statut des partenaires : "Finalement, il s'agit d'une profonde redistribution des relations entre l'accord de branche et le règlement d'entreprise.'.

\section{L'ouverture syndicale}

À deux reprises, les syndicats lancent des initiatives susceptibles de relancer la négociation collective, la première en 1994 limitée à une entreprise, Volkswagen, la seconde, peut-être la plus importante, en novembre 1995, prenant la forme d'un proposition de Pacte pour l'emploi.

De l'accord passé dans l'entreprise au titre de la première négociation, on retiendra la décision de privilégier l'emploi aux dépends de ce qui a constitué le cour de l'action syndicale, durant les décennies passées, les augmentations salariales. L'accord stipule, en effet, que la durée hebdomadaire travaillée s'étend maintenant sur 4 jours, soit 28,8 heures en échange d'une perte de salaires d'environ $10 \%$ et du maintien pendant deux ans de l'état actuel du personnel, sans licenciement.

Le Pacte pour le travail (Bündnis für Arbeit) quant à lui, va plus loin dans la question de l'emploi puisqu'il met en parallèle renoncement aux augmentations de salaires et emploi des chômeurs. L'originalité de la proposition lancée par le président de l'IG Metall au cours de la $18 \mathrm{e}$ session du syndicat de la métallurgie tient en effet à l'offre faite au patronat et à l'État de renoncer pendant trois ans aux augmentations de salaires dans les branches de la métallurgie, en échange de quoi le patronat s'engagerait à créer 300000 emplois et à embaucher 30000 chômeurs de longue durée. L'objectif poursuivi par IG Metall en la matière est triple : reprendre l'offensive en matière de négociation collective, compte tenu des reproches incessants faits aux syndicats de participer par leurs revendications à la croissance des faillites et du chômage. Prendre acte, en second lieu, du mouvement de dérégulation qui affecte les relations professionnelles en l'anticipant ${ }^{2}$. En faisant cette ouverture, le responsable de la métallurgie entend placer non seulement l'État devant l'obligation d'énoncer son plan pour l'emploi, notamment celui concernant les chômeurs de longue durée, mais implique également le patronat, en le forçant à se positionner activement dans le débat politique de l'emploi. Il s'agit ici de définir le syndicat comme défenseur des intérêts des chômeurs et, ce faisant, de recréer le lien de la solidarité au fondement de l'action syndicale. Enfin, devant la dérégulation, qui affecte plus généralement les entreprises européennes confrontées à la globalisation de l'économie, le responsable de l'IG Metall entend défendre la vision d'un syndicalisme au niveau européen, appuyé sur des conseils d'entreprise dotés de fortes prérogatives, décidés à ne pas laisser s'implanter un capitalisme de marché sauvage, avec des syndicats sans

1. Jbid., p. 129.

2. La politique tarifaire cela signifie que l'avenir de la négociation collective réclame des réponses plus différenciées et plus flexibles, ou alors nous scierions la branche sur laquelle nous sommes assis. (Zwickel (K.), Grundsatzreferat 18 ordentlicben Gewerkschaftstag, 10 novembre 1995, p. 15). 
influence et des managers du social dociles*, déjà trop visible. Ainsi en modifiant le contenu de la négociation, IG Metall entend a la fois renouveler le jeu institutionnel entre les partenaires composant ale cœur du système, et réamorcer la mobilisation collective, raison d'être du syndicalisme.

Le pari fait par le responsable IG Metall comporte plusieurs risques, dénoncés tant par les membres du syndicat que par les employeurs. Certains, parmi les premiers, ont mis en avant le danger de se substituer à l'État en matière de politique d'emploi, ce qui est contradictoire avec la stratégie d'un syndicalisme entendu comme strict lieu de la défense des intérêts des salariés. Cela posé, il convient d'ajouter que cette proposition a reçu un large accord au sein des syndicats, et notamment l'entier soutien de la confédération DGB. Quant à certains membres du patronat, ils n'ont pas hésité à réfuter la proposition faite au motif qu'il ne convient pas de se lier les mains en acceptant de créer 330000 emplois lorsque le blocage des salaires suffit tout juste à maintenir les entreprises en l'état. À leurs yeux, la négociation doit avoir lieu sans préalables, étant entendu que pour d'autres représentants patronaux la proposition de cesser toute revendication en matière de hausse des salaires mérite toutefois un sérieux examen ${ }^{1}$. Quant à l'État, il a par la bouche du ministre de l'Économie fait savoir qu'il réfutait, dans un premier temps, de se voir fixer par l'organisation syndicale de la métallurgie les grandes lignes de son plan pour l'emploi, tout en reconnaissant, lui aussi, qu'il ne convenait pas de l'écarter complètement. Enfin, plusieurs économistes se sont déclarés peu convaincus par l'égalité posée par la proposition entre réduction salariale et création d'emploi, au motif qu'il est très difficile de quantifier les créations de cet ordre. D'autres ont craint le coût en termes de solidarité, au motif qu'un chômeur de longue durée est d'une moindre productivité qu'un jeune, d'où il résulte un gain économique largement improbable ${ }^{2}$. Toutefois, il est remarquable de voir combien les positions ont largement évolué, compte tenu des données rendues publiques faisant état de l'aggravation de la situation de l'emploi, des perspectives négatives pour 1996 et des soutiens apportés par plusieurs personnalités, tant politiques, qu'économiques $^{3}$. Le 24 janvier s'est tenu, sous l'autorité du chancelier Kohl, un sommet réunissant les principaux représentants du gouvernement, du patronat et des syndicats, au terme duquel un document de huit pages a été signé, intitulé *Pacte pour l'emploi et pour le maintien de la compétitivité allemande». En échange d'engagements de modération salariale, de flexibilité en matière de durée du travail et de réduction des heures supplémentaires de

1. Pour le patron des patrons, il s'est agi de faire du neuf avec du vieux. (Handelsblatt, 3 janvier 1996). Selon ce dernier, le mouvement de rationalisation n'est pas achevé et les réductions de personnel sont encore nécessaires pour abaisser les couts du travail. Si la branche peut espérer une croissance de la productivité de $6,5 \%$, néanmoins ces gains seront absorbés par une croissance des augmentations salariales de l'ordre de $6 \%$. Comme, de surcroît, les réductions de personnel devraient cette année affecter 120000 postes (dont 65000 situés à l'Ouest), la création demandée par IG Metall porterait le nombre de créations d'emploi à 230000 , ce qui est jugé totalement irréaliste. Pour le responsable de la métallurgie en Basse Saxe, cela ne s'explique que pour des raisons populistes. (Handelsblatt, 6 novembre 1995). Les 17 responsables de la métallurgie l'ont suivi.

2. Die Zeit, 10 novembre 1995.

3. Au premier rang d'entre eux, le ministre du Travail, qui a regretté que les employeurs ne s'emparent pas positivement de cette proposition (Handelsblatt, 20 novembre 1995 et 29-30 décembre 1995), rejoint en cela par le président de la Fondation Friedrich Ebert (Handelsblatt, 7 novembre 1995). Il en va également du député Ost de la CDU, chef de la commissión économique du Bundestag, pour lequel la discipline proposée par IG Metall est la seule issue possible si l'Allemagne entend créer d'ici l'an 2000 les 5 millions de places dont elle a besoin (Handelsblatt, 3 janvier 1996). 
la part des syndicat, le gouvernement a marqué sa volonté de réduire le chômage de moitié d'ici l'an 2000, d'augmenter les places d'apprentissage, mais également de limiter certains avantages acquis, notamment sociaux ${ }^{1}$.

Au delà des appréciations portées sur cette proposition et des stratégies qu'elle est susceptible d'entraîner, son examen nous conduit à nous interroger sur la capacité des acteurs de la négociation tarifaire à innover, à la fois en modifiant son contenu, c'est-à-dire pour l'acteur syndical en réduisant ses marges en termes de gains salarial, tout en cherchant à conserver l'essentiel du jeu, c'est-à-dire un jeu qui permet en même temps la poursuite du système par la réaffirmation du niveau pertinent de la branche, le renouvellement de l'organisation grâce à une représentation des intérêts des adhérents, et la flexibilité requise au niveau de l'adaptation à l'entreprise. Comme on l'a dit plus haut, l'unification a approfondi la fracture entre, d'une part, la logique de participation au jeu institutionnel qui confère au syndicat sa légitimité sociale, le posant comme acteur responsable et partie influente de l'équilibre sociétal, et d'autre part, la logique de mobilisation, qui renvoie à sa légitimité interne reposant sur l'adhésion des membres à l'organisation défendant leurs intérêts catégoriels. On a vu comment la poursuite de cette fracture conduisait à une dérégulation profonde, à une fuite des adhérents, à un blocage du jeu. L'innovation, de ce fait, tient à la capacité de faire reposer sur la solidarité avec les exclus du travail la notion de participation au jeu institutionnel, autrement dit à retrouver les traditions d'un syndicalisme fondé sur la communauté pour mieux refonder l'échange social. À cet égard, dans leur recherche du renouvellement du syndicalisme à travers la crise actuelle, plusieurs acteurs n'hésitent pas à revendiquer l'élargissement des différente forces sociales a la formation d'un pacte regroupant les partis, les églises, les mouvements sociaux ${ }^{2}$. Loin d'accepter de faire correspondre à la reconnaissance de la réalité de la globalisation mondiale des échanges économiques la disparition de la pertinence des lieux concrets du travail, les syndicalistes allemands réclament que soient revalorisées l'entreprise, comme lieu du conflit, et la branche comme lieu de la négociation.

Le cas est-allemand a représenté un cas limite en matière de disjonction des termes de l'autonomie tarifaire reposant sur l'équilibre négocié de la croissance de la productivité du travail et de celle des salaires. Au nom de l'égalisation des conditions de vie et de travail des deux communautés allemandes, ce principe a été négligé, et ce faisant les formes de la cohésion sociales ont été mises à mal, entraînant des coûts considérables. Ils ont porté, d'un côté, sur le contenu de la négociation ultérieure, dans la mesure où celleci s'est trouvée modifiée en raison de la prise en charge de catégories prioritaires telles que l'emploi, en échange de l'acceptation par les partenaires de dérégulations partielles, au niveau de l'entreprise. De l'autre côté, ces coûts ont contraint les acteurs de la négociation professionnelle à redéfinir leur capacité à poursuivre le jeu institutionnel, en renouvelant les base de la mobilisation de leurs adhérents. Sur ce point, l'expérience estallemande a servi de révélateur à la crise profonde qui, depuis 1993, a remis à l'ordre du jour les questions soulevées antérieurement sur la pertinence de la 
branche comme lieu de la négociation. En favorisant la signature de clauses d'exception, ou la mise en place de règlements particuliers à certaines entreprises, elle aura révélé autant les tensions qui pèsent sur ce système, que sa capacité de flexibilité et d'innovation, notamment en ce qui concerne les acteurs syndicaux.

Nombreux sont les acteurs - étatiques, syndicaux ou patronaux - qui sont conscients de l'intérêt du maintien du système de relations professionnelles faisant reposer l'homogénéité des groupes sur la limitation des inégalités et sur la participation collective de leurs membres. Les syndicats y ont, en effet, intérêt dans la mesure où les augmentations salariales garanties au niveau d'une branche favorisent une homogénéité territoriale. Les employeurs, tout autant, dans la mesure où des conventions de branche interdisent toute forme de dumping salarial, en assurant de surcroît la paix sociale. L'État, enfin, y trouve également son intérêt dans la mesure où il ne lui revient pas d'assumer les compétences dont il ne dispose pas, en matière de gestion du marché du travail, et qu'aucun des autres partenaires sociaux ne souhaiterait d'ailleurs lui conférer ${ }^{1}$. L'abaissement des négociations au niveau de l'entreprise aurait ainsi des effets négatifs très importants, en matière de cohésion sociale et d'intégration des intérêts. Les entreprises les plus puissantes disposeraient certes davantage de capacités de redistribution pour ses membres, mais en laissant les moins dotées s'enfoncer dans la faiblesse et l'absence de représentation ${ }^{2}$.

À cet égard, les risques qui menacent l'institution paritaire allemande sont grands, en particulier à la suite des transformations du jeu économique mondial. Pour un observateur comme Wolfgang Streeck, le plus grand danger réside, en effet, dans le mouvement de globalisation qui affecte les échanges économiques, rendant obsolètes les formes de direction économique fondées sur l'intervention publique et la négociation étatique, au profit des régulations reposant presque exclusivement sur les unités privées ${ }^{3}$. Inexportable et intransférable hors des frontières allemandes, le mode de régulation sociale serait ainsi la victime toute désignée d'une dérégulation économique non maîtrisée. L'enjeu du maintien du système de régulation corporatiste à l'allemande semble ainsi désormais résider dans la capacité de ses acteurs à trouver le point d'équilibre délicat entre régulation collective, flexibilité au niveau de l'entreprise et représentation des intérêts individuels. En raison de la pluralisation des intérêts représentés, ce sont autant le contenu des objets de la négociation paritaire que la nature des stratégies des organisations qui sont appelés à évoluer profondément.

1. Hege (A.), Allemagne, conflictualité contenue, maîtrisée, efficace ?•, IRES. Cbronique Inemationale, 33, 1995.

2. Lepsius (R.), -Die gegenwärtige Diskussion zur Situation des Sozialstaats in Deutschland., Jabrbucb für Arbeit + Tecbnik, 1995.

3. Streeck (W.), -Der deutsche Kapitalismus : Gibt es ihn ? Kann er überleben ?., Interessenvertretung, Organisationsentwicklung und gesellscbaftsreform, Gewerkscbafts - und Gesellschaftspolitiscbes Forum der IG Metall, 15-16 juin 1995. 